\title{
Time requirements in closed and open batch distillation arrangements for separation of a binary mixture
}

\author{
Shuo Zhao ${ }^{1,2 *}$, Peng Bai ${ }^{1,2}$, Xianghai Guo ${ }^{1,2}$, Ke Tang, ${ }^{1,2}$ Guangzhong $\mathrm{Li}^{1,2}$ \\ ${ }^{1}$ Tianjin University, School of Chemical Engineering and Technology, Tianjin 300072, P.R. China \\ ${ }^{2}$ Tianjin University, Key Laboratory of Systems Bioengineering, Ministry of Education, Tianjin 300072, P.R. China \\ *Corresponding author: e-mail: shuozhao@tju.edu.cn
}

\begin{abstract}
Batch time requirements are provided for the separation of binary zeotropic mixtures in two different multivessel columns (with and without vapor bypass), a non-cyclic two-vessel column and a regular batch column based on dynamic simulations. The first three columns are operated as closed (total reflux) systems and the regular batch column is operated as an open (partial reflux) system. We analyze the effects of feed composition, relative volatility and product specification on the time requirements. The multivessel arrangements perform better than the regular batch column, which requires from 4.00 to $34.67 \%$ more time to complete a given separation. The elimination of the vapor bypass in the multivessel column is impractical though it has a positive effect on the batch time requirements. Thus, the multivessel column, with the vapor stream bypassing the intermediate vessel, is proposed as the best candidate for a binary zeotropic mixture with low concentration of light component, low relative volatility and high product purity demand. Furthermore, an experimental multivessel column with vapor bypass is built and the corresponding experiments verify the simulations.
\end{abstract}

Keywords: time requirements, closed operation, multivessel column, binary mixture.

\section{INTRODUCTION}

The regular batch column operated as an open system (with continuous product withdrawal) is widely used in the fine and specialty chemical and pharmaceutical industries, as shown in Figure 1(a). The feed is charged to the reboiler and the products are taken from the top of the column sequentially one after the other during a rectification process. Moreover, in the past two decades, batch distillation has aroused increasing interest because of its own advantages such as simplicity of operation, flexibility, less capital investment, etc. With an increasing demand for high quality products and flexible plants, the researches of batch distillation have mainly focused on non-conventional operation modes, such as closed (total reflux) operations, and novel batch column configurations, like inverted column ${ }^{1-3}$, middle-vessel column ${ }^{4-13}$ and multivessel column.

The closed operation is one of the promising modes of batch distillation, which has several main advantages over the conventional partial reflux modes such as achieving the maximum fractionating capacity, easier operational control and less disturbances to product quality and yield from the practical point of view ${ }^{14,15}$. In addition, closed operation modes are preferable in terms of separation time or energy requirements ${ }^{16-20}$. The non-cyclic two-vessel column, shown in Figure 1(b), is a simple configuration of the closed operation, which means that there is no distillate or bottom stream out from the column and the final products are accumulated in the condenser vessel and the reboiler when the specifications are satisfied ${ }^{21,22}$.

Multivessel column, as a novel batch distillation configuration, can be viewed as a generalization of previously proposed batch distillation schemes, including the inverted column and the middle-vessel column. With $n$ vessels along the column including a reboiler, a condenser vessel, and $n-2$ intermediate vessels, it is possible to obtain $n$ pure products in the multivessel column in a single batch. In most cases, the term is used for processes with at least four product vessels including the reboiler and the distillate receiver. Moreover, the main reason for using the arrangement is that it usually requires less energy or shorter batch time for a given heat input than a regular batch distillation column ${ }^{23-31}$.

To the best of our knowledge, there is still no result for separating a binary zeotropic mixture by the multivessel column. In this paper, it is the first time to use the multivessel column to separate a binary zeotropic mixture. For designing the multivessel column, two configurations are developed. One is the conventional multivessel column, in which the vapor stream from the lower section bypasses the intermediate vessel and enters the upper section, as shown in Figure 1(c). The other is the modified multivessel column, where both the liquid and the vapor streams enter the intermediate vessel, as illustrated in Figure 1(d). Furthermore, we compare the two different multivessel column configurations with the non-cyclic two-vessel column and the regular batch column in terms of time requirements for the separation of a binary zeotropic mixture. Two multivessel columns and the non-cyclic two-vessel column are carried out in closed mode, and the regular batch column is operated under constant reflux ratio. Meanwhile, the effect of feed composition, relative volatility and product specification on the time requirements is also studied based on dynamic simulations of the processes. A laboratory-scale multivessel column with the vapor stream bypassing the intermediate vessel is built, and then the corresponding experiments verify the simulations.

\section{New Operation Process}

As one of the most promising new batch column configurations, the multivessel column is capable of separating a dual composition mixture with constant total reflux. The operation mode of the multivessel column configurations for separating a binary zeotropic mixture is illustrated in Figures 1(c) and 1(d). 


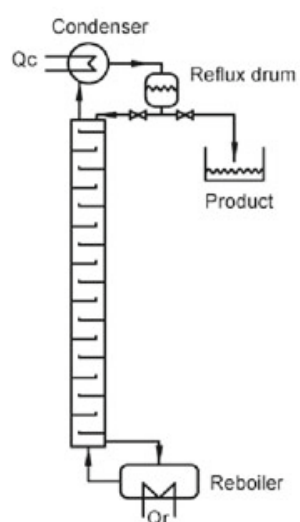

(a)

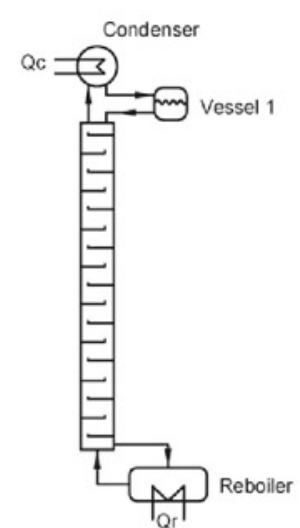

(b)

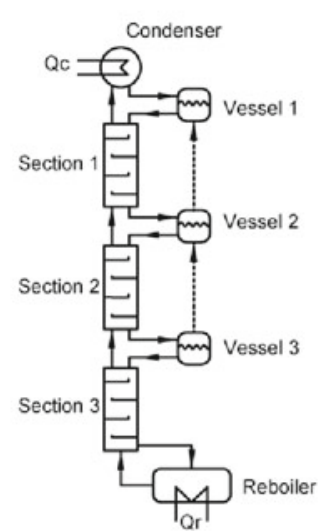

(c)

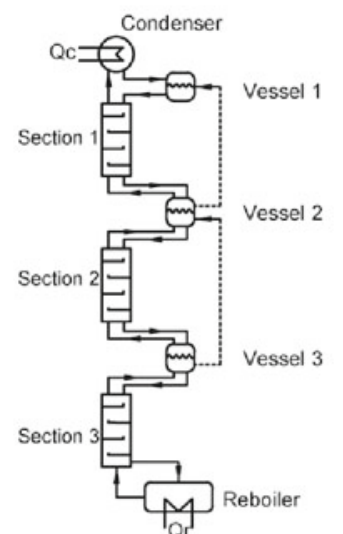

(d)

Figure 1. (a) Regular batch column; (b) non-cyclic two-vessel column; (c) conventional multivessel column with vapor bypass; (d) modified multivessel column without vapor bypass

There are 3 vessels which can be connected to or isolated from the column by adjusting valves in the system. Moreover, for each vessel, a corresponding liquid level controller is designed. The Vessel 1 (reflux drum) is connected to the condenser as the product receiver. At the beginning of the operation, all the vessels are connected to the column and filled with a specified amount of feed charge. The total reflux operation starts and goes on until the holdup in the top drum reaches its on-specification composition for the first time. At this moment, all of the filled vessels are isolated from the column, the liquid in Vessel 1 is drained away as the top product and the holdups in other 2 vessels are moved upwards in turn through switching the pumps and valves in the pipelines. Consequently, the present holdups in Vessel 1 and Vessel 2 are the previous holdups in Vessel 2 and Vessel 3 respectively, while Vessel 3 is empty at present and will not be connected to the column any more. Then, the remaining filled vessels (Vessel 1 and Vessel 2) are connected to the column and the system repeats the total reflux operation. When the concentration in Vessel 1 reaches its qualification for the second time, the remaining two vessels are isolated from the system, the liquid in Vessel 1 is still drained away as the top product and the holdup in Vessel 2 is shifted to Vessel 1. And then, the column with only one vessel (Vessel 1) is operated under total reflux once again. The holdup in Vessel 1 is discharged as product after its concentration meets the requirement for the third time. The whole operation process is carried out with 3 total reflux operations until the holdups in all the vessels are drained away as the top satisfactory product. The number of vessels connected to the column is reduced by one after each closed operation. Hence, the constant total reflux operation is characterized by retaining infinite reflux ratio and gradually reducing the number of vessels connected to the column during the whole batch operation. Moreover, it is well known that the total reflux mode can achieve the maximum fractionating capacity. Therefore, the novel operation mode has the higher efficiency than the partial reflux mode.

\section{SIMULATIONS}

\section{The mathematical models}

The mathematical models used in our simulations consist of material balances, vapor-liquid equilibriums, and summation equations. Before proceeding, we present some simplifying assumptions as follows: (1) constant molar vapor flows (in which energy balance is neglected); (2) constant molar liquid holdup on all stages (in which liquid flow dynamics are neglected); (3) constant relative volatility; (4) negligible vapor holdup; (5) perfect mixing and equilibrium in all stages and in all vessels; (6) constant pressure; (7) constant tray efficiency $(100 \%)$; (8) total condensation without sub-cooling. Then, the models of the conventional and modified multivessel column are obtained. These assumptions are introduced to simplify the model, and similar results can be obtained when the assumptions are relaxed.

\section{The model of the conventional multivessel column}

The conventional multivessel column is modeled as a stack of stages (counted from the top) as shown in Figure 2. Index $i$ denotes the stages (including the theoretical stages and the vessels), and index $j$ denotes the components. Note that the vapor flow $V$ does not pass through the intermediate vessels, so these are not counted as part of the theoretical stages. Hence, the material balances can be written as follows:

The top vessel $(i=1)$

$H_{i} \frac{d x_{i, j}}{d t}=V_{i+1} y_{i+1, j}-L_{i} x_{i, j}$

Column stage $i$ away from the intermediate vessels

$H_{i} \frac{d x_{i, j}}{d t}=L_{i-1} x_{i-1, j}+V_{i+1} y_{i+1, j}-L_{i} x_{i, j}-V_{i} y_{i, j}$

As shown in Figure 2(a), when the intermediate vessel $i$ is connected to column, the material balance equation for column stage $i-1$ above the intermediate vessel $i$ is

$H_{i-1} \frac{d x_{i-1, j}}{d t}=L_{i-2} x_{i-2, j}+V_{i+1} y_{i+1, j}-L_{i-1} x_{i-1, j}-V_{i-1} y_{i-1, j}$

For intermediate vessel $i$, the material balance equation is

$H_{i} \frac{d x_{i, j}}{d t}=L_{i-1} x_{i-1, j}-L_{i} x_{i, j}$ 


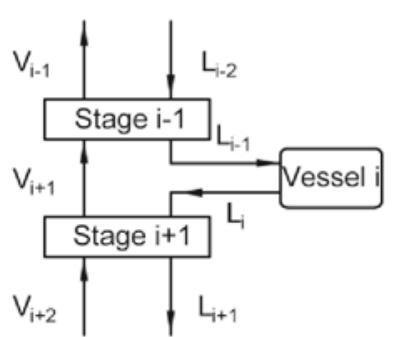

(a)

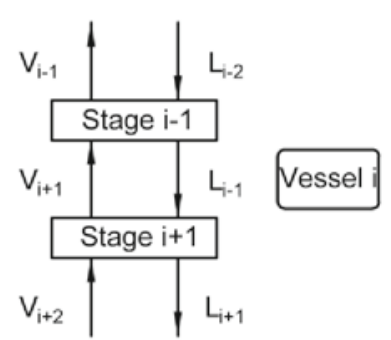

(b)
Figure 2. Vapor and liquid flow state in the conventional multivessel column and vessels (a) the $i$ th vessel connected to column; (b) the ith vessel moved upwards

For column stage $i+1$ under the intermediate vessel $i$, the material balance equation is

$H_{i+1} \frac{d x_{i+1, j}}{d t}=L_{i} x_{i, j}+V_{i+2} y_{i+2, j}-L_{i+1} x_{i+1, j}-V_{i+1} y_{i+1, j}$

As shown in Figure 2(b), when the intermediate vessel $i$ is isolated from column, the material balance equation for column stage $i-1$ above the intermediate vessel $i$ is

$H_{i-1} \frac{d x_{i-1, j}}{d t}=L_{i-2} x_{i-2, j}+V_{i+1} y_{i+1, j}-L_{i-1} x_{i-1, j}-V_{i-1} y_{i-1, j}$

For column stage $i+1$ under the intermediate vessel $i$, the material balance equation is

$H_{i+1} \frac{d x_{i+1, j}}{d t}=L_{i-1} x_{i-1, j}+V_{i+2} y_{i+2, j}-L_{i+1} x_{i+1, j}-V_{i+1} y_{i+1, j}$

For the reboiler $\left(i=n_{\mathrm{N}}+n_{\mathrm{V}}\right)$, the corresponding balance equation is given by

$\frac{d H_{B} x_{i, j}}{d t}=L_{i-1} x_{i-1, j}-V_{i} y_{i, j}$

The material balance equations of the conventional multivessel column consist of Eqs. (1)-(8). The additional relations for solving the model equations are as follows:

The vapor-liquid equilibrium

$y_{i, j}=\frac{\alpha x_{i, j}}{1-(\alpha-1) x_{i, j}}$

The summation equation

$\sum_{j=1}^{n_{C}} x_{i, j}=\sum_{j=1}^{n_{C}} y_{i, j}=1$

\section{The model of the modified multivessel column}

The modified multivessel column is designed as a stack of stages (counted from the top) as shown in Figure 3. Index $i$ denotes the stages (including the theoretical stages and the vessels), and index $j$ denotes the components.

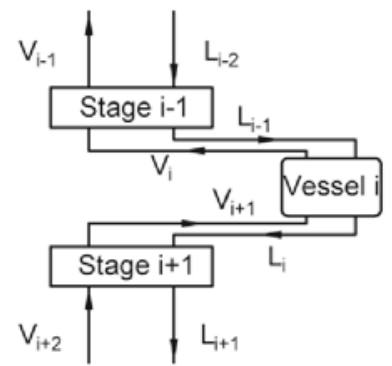

(a)

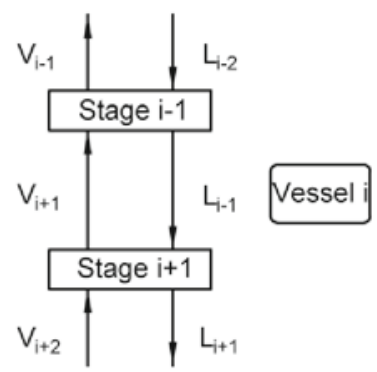

(b)
Figure 3. Vapor and liquid flow state in the modified multivessel column and vessels (a) the $i$ th vessel connected to column; (b) the ith vessel moved upwards
Note that unlike the conventional multivessel column, both of the vapor flow $V$ and liquid flow $L$ pass through the intermediate vessels, so these should be counted as part of the theoretical stages having larger holdup. Thus, the material balances can be written as follows:

The top vessel $(i=1)$

$H_{i} \frac{d x_{i, j}}{d t}=V_{i+1} y_{i+1, j}-L_{i} x_{i, j}$

Column stage $i$ away from the intermediate vessels

$H_{i} \frac{d x_{i, j}}{d t}=L_{i-1} x_{i-1, j}+V_{i+1} y_{i+1, j}-L_{i} x_{i, j}-V_{i} y_{i, j}$

As shown in Figure 3(a), when the intermediate vessel $i$ is connected to column, the material balance equation for the intermediate vessel $i$ is given by

$H_{i} \frac{d x_{i, j}}{d t}=L_{i-1} x_{i-1, j}+V_{i+1} y_{i+1, j}-L_{i} x_{i, j}-V_{i} y_{i, j}$

As shown in Figure 3(b), when the intermediate vessel $i$ is isolated from column, the material balance equation for column stage $i-1$ above the intermediate vessel $i$ is rewritten as

$H_{i-1} \frac{d x_{i-1, j}}{d t}=L_{i-2} x_{i-2, j}+V_{i+1} y_{i+1, j}-L_{i-1} x_{i-1, j}-V_{i-1} y_{i-1, j}$

Column stage $i+1$ under the intermediate vessel $i$, the material balance equation is

$H_{i+1} \frac{d x_{i+1, j}}{d t}=L_{i-1} x_{i-1, j}+V_{i+2} y_{i+2, j}-L_{i+1} x_{i+1, j}-V_{i+1} y_{i+1, j}$

For the reboiler $\left(i=n_{\mathrm{N}}+n_{\mathrm{V}}\right)$, the corresponding balance equation is given by

$\frac{d H_{B} x_{i, j}}{d t}=L_{i-1} x_{i-1, j}-V_{i} y_{i, j}$

Therefore, we obtain the material balance equations of the modified multivessel column which consist of Eqs. (11)-(16). Moreover, the vapor-liquid equilibrium equation and the summation equation are the same as those in the model of the conventional multivessel column.

The dynamic mathematical model of the multivessel column takes the form of a set of differential and algebraic equations (DAE system) with a total of $\left(n_{\mathrm{C}}+1\right) \times\left(n_{\mathrm{N}}+n_{\mathrm{V}}\right)$ state variables, where $n_{C}$ is the number of components, $n_{N}$ is the total number of stages in the column sections and $n_{V}$ is the number of vessels (two for the regular column and the non-cyclic two-vessel column, and four for the multivessel configurations). In addition, the simple models for the regular batch column under constant reflux and the non-cyclic two-vessel column under total reflux are taken from Mujtaba ${ }^{32}$.

The DAE system is implemented by using the MATLAB software with Runge-Kutta integral method. Furthermore, the solver ODE45 in MATLAB software is proved to be very efficient in initializing the simulations in all cases and no problems are experienced on this issue.

\section{Simulation aspects}

Batch time comparisons are provided for the separation of a binary zeotropic system. Specifications for four batch column configurations are shown in Table 1. The initial value of purity in each column is the same as the feed concentration. The batch time calculations do not include charging of the column and heating up the column and feed mixture to the boiling temperature. 
Table 1. Column data

\begin{tabular}{|l|c|c|c|c|}
\hline & Conventional multivessel column & Modified multivessel column & Regular batch column & Non-cyclictwo-vessel column \\
\hline Stages per section & $N_{1}=10 ; N_{2}=10 ; N_{3}=10$ & $N_{1}=10 ; N_{2}=10 ; N_{3}=10$ & $N_{\text {total }}=30$ & $N_{\text {total }}=30$ \\
\hline Number of vessels & $\begin{array}{c}1 \text { condenser vessel; } \\
\text { intermediate vessels; } \\
1 \text { reboiler vessel }\end{array}$ & $\begin{array}{c}1 \text { condenser vessel; } \\
\text { intermediate vessels; } \\
1 \text { reboiler vessel }\end{array}$ & $\begin{array}{c}1 \text { reflux drum; } \\
\text { reboilervessel }\end{array}$ & $\begin{array}{c}1 \text { condenser vessel; } \\
1 \text { reboiler vessel }\end{array}$ \\
\hline Initial feed & $F=200 \mathrm{~mol}$ & $F=200 \mathrm{~mol}$ & $F=200 \mathrm{~mol}$ & $F=200 \mathrm{~mol}$ \\
\hline Stage holdup & $H_{\mathrm{i}}=0.1 \mathrm{~mol}$ & $H_{\mathrm{i}}=0.1 \mathrm{~mol}$ & $H_{\mathrm{i}}=0.1 \mathrm{~mol}$ & $H_{\mathrm{i}}=0.1 \mathrm{~mol}$ \\
\hline Vapor flow & $V=2 \mathrm{~mol} / \mathrm{min}$ & $V=2 \mathrm{~mol} / \mathrm{min}$ & $V=2 \mathrm{~mol} / \mathrm{min}$ & $V=2 \mathrm{~mol} / \mathrm{min}$ \\
\hline Product recovery & $R e c=75 \%$ & $R e c=75 \%$ & $R e c=75 \%$ & $R e c=75 \%$ \\
\hline
\end{tabular}

These are considered to be the same for both of the multivessel column configurations, the non-cyclic two-vessel column and the regular batch column. Especially, the time to spend in shifting the holdup from lower vessel to the upper vessel is also neglected in simulations of the multivessel column configurations. During the closed operation period, the reflux flow rate has a constant value which is the same as the vapor flow rate. As a result, the holdups in all the vessels and in the reboiler are constant as their predetermined values throughout the closed operation process. By considering the amount of feed, feed composition and product specifications (including the recovery and the purity of the product), the holdup of each vessel in both of the multivessel configurations can be calculated in advance. The values of the reflux ratio in regular batch column are determined in order to satisfy the product recovery and the product purity simultaneously. The liquid rate in the open operation also has a constant value which is determined by the vapor flow rate and reflux ratio. In addition, it should be noted that in practice, the simulations are carried out until some criterions are met, e.g. the light component concentration in each vessel fulfills the product purity requirement and the product recovery in the vessels reaches $75 \%$.

The effect of liquid holdup on the column stages in the separation times is not addressed in this study. All column configurations have constant and very small liquid holdup negligible compared to the initial feed $(1.5 \%$ of the charge). In practice, this means that almost all the initial charge is recovered in the vessels at the end of the process. It also means that the dynamics inside the column sections are negligible compared to that in the vessels and the change of the light component concentration in each vessel is almost instantaneous. Note that the dynamics inside the column sections mean the dynamic changes of vapor phase composition and liquid phase composition on each stage in the column.

\section{SIMULATION RESULTS AND DISCUSSION}

Conventional multivessel column versus regular batch column and non-cyclic two-vessel column

From the batch time comparisons in Table 2, it is clear that when feed composition $\left(x_{\mathrm{F}}\right)$ is less than 0.5 , the co-

Table 2. Batch time calculations of different column configurations for different operating conditions

\begin{tabular}{|c|c|c|c|c|c|c|c|c|}
\hline \multicolumn{3}{|c|}{ Conditions } & \multicolumn{2}{|c|}{ Conventional multivessel column } & \multicolumn{2}{|c|}{ Regular batch column } & \multicolumn{2}{|c|}{ Non-cyclic two-vessel column } \\
\hline$x_{F}$ & $\alpha$ & $x_{P}$ & $\begin{array}{c}H v \\
{[\mathrm{~mol}]}\end{array}$ & Batch time [min] & $R$ & Batch time [min] & $\begin{array}{c}H_{\top} \\
{[\mathrm{mol}]}\end{array}$ & Batch time [min] \\
\hline \multirow{3}{*}{0.15} & \multirow{3}{*}{3.0} & 0.99 & 7.6 & 81.9 & 6.2 & 87.7 & 22.7 & 102.0 \\
\hline & & 0.995 & 7.5 & 86.7 & 6.5 & 91.5 & 22.6 & 113.3 \\
\hline & & 0.999 & 7.5 & 102.2 & 6.9 & 95.6 & 22.5 & 139.9 \\
\hline \multirow{3}{*}{0.15} & \multirow{3}{*}{2.5} & 0.99 & 7.6 & 103.4 & 8.7 & 118.2 & 22.7 & 123.5 \\
\hline & & 0.995 & 7.5 & 106.1 & 9.0 & 121.1 & 22.6 & 135.9 \\
\hline & & 0.999 & 7.5 & 122.3 & 9.6 & 127.4 & 22.5 & 165.4 \\
\hline \multirow{3}{*}{0.15} & \multirow{3}{*}{2.0} & 0.99 & 7.6 & 150.1 & 13.4 & 170.5 & 22.7 & 168.7 \\
\hline & & 0.995 & 7.5 & 152.8 & 14.2 & 180.4 & 22.6 & 182.2 \\
\hline & & 0.999 & 7.5 & 165.9 & 15.5 & 196.9 & 22.5 & 218.6 \\
\hline \multirow{3}{*}{0.15} & \multirow{3}{*}{1.5} & 0.99 & 7.6 & 299 & 31.7 & 386.5 & 22.7 & 313.4 \\
\hline & & 0.995 & 7.5 & 306.5 & 35.0 & 425.5 & 22.6 & 338.0 \\
\hline & & 0.999 & 7.5 & 361.6 & 46.0 & 553.5 & 22.5 & 398.3 \\
\hline \multirow{3}{*}{0.3} & \multirow{3}{*}{1.5} & 0.99 & 15.2 & 297.0 & 14.2 & 354.0 & 45.5 & 368.0 \\
\hline & & 0.995 & 15.1 & 320.6 & 15.3 & 378.8 & 45.2 & 415.7 \\
\hline & & 0.999 & 15.0 & 380.9 & 18.2 & 444.0 & 45.0 & 528.3 \\
\hline \multirow{3}{*}{0.5} & \multirow{3}{*}{1.5} & 0.99 & 25.3 & 339.4 & 7.2 & 316.7 & 75.8 & 500.4 \\
\hline & & 0.995 & 25.1 & 377.5 & 7.8 & 340.5 & 75.4 & 577.1 \\
\hline & & 0.999 & 25.0 & 478.4 & 9.1 & 387.9 & 75.1 & 758.0 \\
\hline \multirow{3}{*}{0.75} & \multirow{3}{*}{1.5} & 0.99 & 37.9 & 379.3 & 3.7 & 273.7 & 113.6 & 581.6 \\
\hline & & 0.995 & 37.7 & 437.3 & 4.0 & 293.9 & 113.1 & 697.6 \\
\hline & & 0.999 & 37.5 & 577.9 & 5.0 & 349 & 112.6 & 968.3 \\
\hline
\end{tabular}


nventional multivessel column needs less operation time and provides higher separation efficiency than the regular batch column and the non-cyclic two-vessel column. As shown in Figure 4, when $x_{\mathrm{F}}$ is 0.15 and relative volatility $(\alpha)$ is 1.5 , compared with the regular batch column and the non-cyclic two-vessel column, the conventional multivessel column saves the batch time by $34.67 \%$ and $9.21 \%$, respectively. Meanwhile, it is important to note that in order to simultaneously satisfy the product recovery $(\operatorname{Rec}=75 \%)$ and the product purity $\left(x_{\mathrm{P}}=0.999\right)$, the regular batch column has to adopt a very high reflux ratio $(R=46.0)$ under this operating condition. Moreover, with the decrease of feed composition and relative volatility, the conventional multivessel column saves more operation time compared to the regular batch column. It means that the feed condition has direct influence on the batch time requirements.

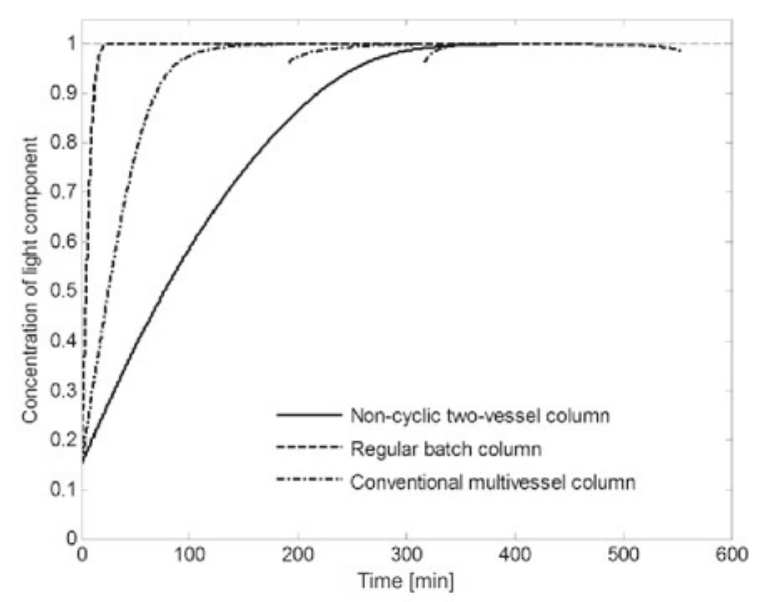

Figure 4. Top concentration profile of different column configurations $\left(x_{\mathrm{F}}=0.15, \alpha=1.5, x_{\mathrm{P}}=0.999\right)$

It is easy to find that the time requirements in all the batch distillation arrangements increase with the augment of the product purity under the certain feed condition in Table 2. Furthermore, the differences between the time requirements of the conventional multivessel column and the regular batch column increase with the augment of the product purity, when $x_{\mathrm{F}}$ is 0.15 and $\alpha$ is less than 2 . This indicates that the conventional multivessel column is more suitable for separation of a binary mixture that is hard to be separated.

However, it can be observed in Table 2 that when $x_{\mathrm{F}}$ is more than 0.5 or $\alpha$ is more than 3.0, the operation time of the regular batch column is less than that of the conventional multivessel column. It means that the multivessel column is not always advantageous.

The above simulation results illustrate that the multivessel configuration has the following advantages. First, compared to the open batch column depending on the partial reflux policy, the multivessel column has lower time requirements and higher separation efficiency for obtaining the desired product. Second, the multivessel column is more reasonable and efficient compared to the non-cyclic two-vessel column. Third, the novel closed batch distillation arrangement is more suitable for a binary zeotropic system that is difficult to be separated, such as separation of a mixture with low concentration of light component, low relative volatility and high product purity demand.

\section{Conventional multivessel column versus modified mul- tivessel column}

From Table 3, it can be seen that the elimination of the vapor bypass in the multivessel column has a significant effect in the batch time requirements. The modified multivessel column requires from 1.18 to $18.33 \%$ less time than the conventional multivessel column, when the $x_{\mathrm{F}}$ is less than 0.5 . Moreover, with the decrease of feed composition and relative volatility, the modified multivessel column saves more operation time compared to the conventional multivessel column. It is also clear that both of the multivessel configurations require more operation time with the increase of the product purity under the certain feed condition. Nevertheless, the differences between time requirements of the two multivessel columns decrease with the increase of the product purity, when $x_{\mathrm{F}}$ is 0.15 and $\alpha$ is less than 2.5 . This indicates that the batch time requirement exhibits a strong dependence on the separation condition, including the feed condition and the product quality demand.

In addition, when $x_{\mathrm{F}}$ is more than 0.5 , the batch time of the modified multivessel column is longer than that of the conventional multivessel column. It shows that the modified multivessel column does not always display superiority over the conventional multivessel column, which further verifies that the multivessel column is more suitable for a binary system with low concentration of light component and low relative volatility.

The significant differences between the two different multivessel arrangements should be attributed to the large holdups in the two intermediate vessels of the modified multivessel column compared to the conventional column. However, the intermediate vessels should not be considered simply as equilibrium stages. The dynamics in the intermediate vessels play a decisive role in the separation time requirements due to the larger holdups in the two intermediate vessels compared to the stages inside the column.

Figure 5 illustrates what is happening in the vessels of the multivessel columns during the process, when $x_{\mathrm{F}}$ is $0.15, \alpha$ is 1.5 and $x_{\mathrm{P}}$ is 0.99 . Figures 5(a) and 5(b) show the composition dynamics of the light component in each vessel for the conventional multivessel column and the modified multivessel column, respectively. From Figure 5, it is shown that the operation process of the conventional multivessel column is greatly delayed, due to the vapor bypass from the lower section to the upper section of the column. Furthermore, we can believe that the light component in the intermediate vessels is depleted in a slow rate because there is no vapor stream coming in contact with the liquid holdup in it. This disadvantage of the conventional multivessel column is removed in the so-called modified multivessel column, where the vapor stream from the lower section enters the intermediate vessels. The elimination of the vapor bypass enhances the composition dynamics in the intermediate vessels, thus the process becomes faster. However, it is obvious that the elimination of the vapor bypass has negligible effect in the composition dynamics of the light component in the reboiler. It is reasonable that since the 
Table 3. Batch time calculations of two multivessel column configurations for different operating conditions

\begin{tabular}{|c|c|c|c|c|c|c|}
\hline \multicolumn{3}{|c|}{ Conditions } & \multicolumn{2}{|c|}{ Conventional multivessel column } & \multicolumn{2}{|c|}{ Modified multivessel column } \\
\hline$x_{F}$ & $\alpha$ & $x_{P}$ & $\begin{array}{c}H_{\mathrm{v}} \\
{[\mathrm{mol}]}\end{array}$ & $\begin{array}{l}\text { Batch time } \\
\text { [min] }\end{array}$ & $\begin{array}{c}H_{v} \\
{[\mathrm{~mol}]}\end{array}$ & $\begin{array}{l}\text { Batch time } \\
\text { [min] }\end{array}$ \\
\hline \multirow{3}{*}{0.15} & \multirow{3}{*}{3.0} & 0.99 & 7.6 & 81.9 & 7.6 & 77.8 \\
\hline & & 0.995 & 7.5 & 86.7 & 7.5 & 83.7 \\
\hline & & 0.999 & 7.5 & 102.2 & 7.5 & 97.7 \\
\hline \multirow{3}{*}{0.15} & \multirow{3}{*}{2.5} & 0.99 & 7.6 & 103.4 & 7.6 & 92.5 \\
\hline & & 0.995 & 7.5 & 106.1 & 7.5 & 99.8 \\
\hline & & 0.999 & 7.5 & 122.3 & 7.5 & 117.7 \\
\hline \multirow{3}{*}{0.15} & \multirow{3}{*}{2.0} & 0.99 & 7.6 & 150.1 & 7.6 & 123.6 \\
\hline & & 0.995 & 7.5 & 152.8 & 7.5 & 131.9 \\
\hline & & 0.999 & 7.5 & 165.9 & 7.5 & 150.1 \\
\hline \multirow{3}{*}{0.15} & \multirow{3}{*}{1.5} & 0.99 & 7.6 & 299 & 7.6 & 244.2 \\
\hline & & 0.995 & 7.5 & 306.5 & 7.5 & 257.9 \\
\hline & & 0.999 & 7.5 & 361.6 & 7.5 & 314.6 \\
\hline \multirow{3}{*}{0.3} & \multirow{3}{*}{1.5} & 0.99 & 15.2 & 297.0 & 15.2 & 284.3 \\
\hline & & 0.995 & 15.1 & 320.6 & 15.1 & 311.1 \\
\hline & & 0.999 & 15.0 & 380.9 & 15.0 & 376.4 \\
\hline \multirow{3}{*}{0.5} & \multirow{3}{*}{1.5} & 0.99 & 25.3 & 339.4 & 25.3 & 344.3 \\
\hline & & 0.995 & 25.1 & 377.5 & 25.1 & 384.4 \\
\hline & & 0.999 & 25.0 & 478.4 & 25.0 & 490.3 \\
\hline \multirow{3}{*}{0.75} & \multirow{3}{*}{1.5} & 0.99 & 37.9 & 379.3 & 37.9 & 383.4 \\
\hline & & 0.995 & 37.7 & 437.3 & 37.7 & 442.6 \\
\hline & & 0.999 & 37.5 & 577.9 & 37.5 & 587.6 \\
\hline
\end{tabular}

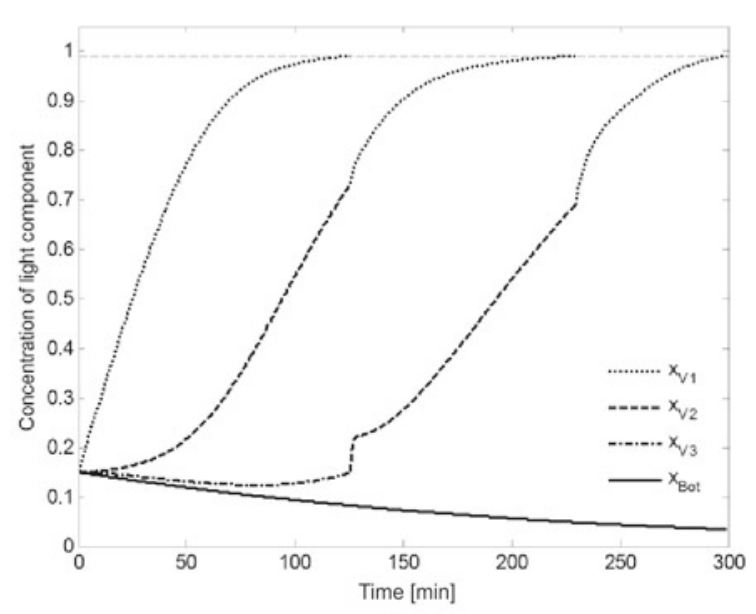

(a)

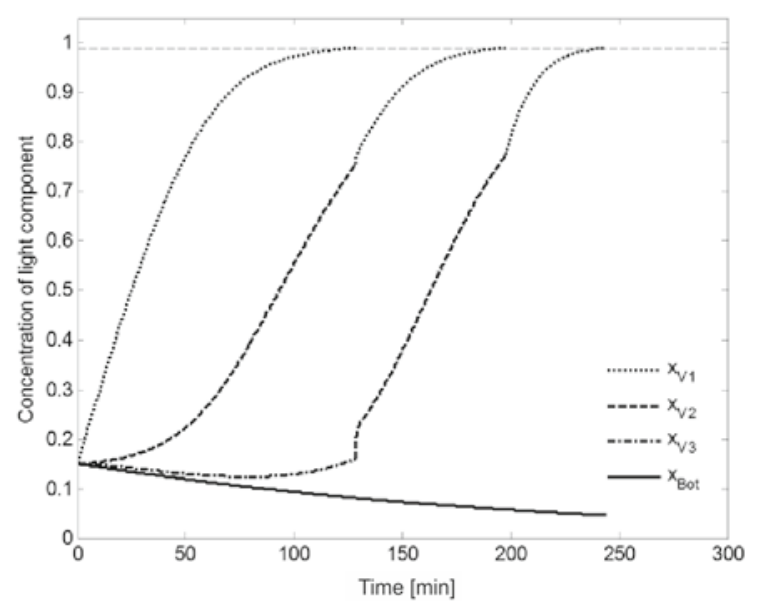

(b)

Figure 5. Concentration of the light component in each vessel for two different multivessel columns (a) Conventional multivessel column; (b) Modified multivessel column

vapor flow out from the reboiler vessel, the liquid flow into the reboiler remain almost unchanged in the two multivessel configurations.

In general, the elimination of the vapor bypass in the multivessel column improves the dynamics in the vessels for separation of a mixture with low concentration of light component and low relative volatility. However, such a modification can give rise to practical problems and is mostly of theoretical interest, at least for the mixtures studied here. Thus, the conventional multivessel column, with the vapor stream bypassing the intermediate ves- sel, is proposed as the best candidate for separation of a binary zeotropic mixture.

\section{THE EXPERIMENTAL SECTION}

\section{Experimental apparatus and procedure}

The laboratory-scale multivessel column with the vapor stream bypassing the intermediate vessel used to verify the proposed operation strategy is shown in Figure 6. It consists of a $1 \mathrm{~L}$ reboiler, a condenser, three packed 


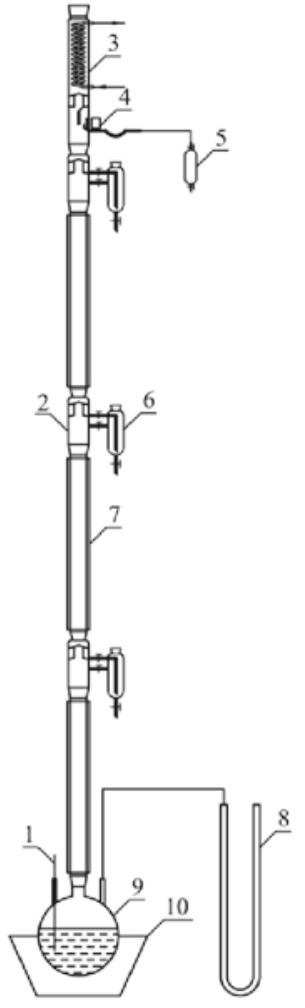

Figure 6. Scheme for the experimental setup (1 - thermocouple; 2 - liquid trap; 3 - condensor; 4 - electromagnet; 5 - product receiver; 6 - vessel; 7 - packed column section; 8 - pressuremeter; 9 - reboiler; 10 - heater)

column sections of 8 theoretical trays and an inner diameter of $30 \mathrm{~mm}$, a product receiver, three liquid traps and three vessels with a maximum holdup of $60 \mathrm{~mL}$. Each column section is packed with $\varphi 3 \times 3 \mathrm{~mm}$ Dixon rings, and the height of each packed layer is $0.5 \mathrm{~m}$.

The initial liquid mixture is fed in along the column and vessels into the reboiler in order to make the concentration uniform, which approximately fulfills the assumption that the initial concentration everywhere in the system is the same. As the total reflux operation proceeds, the concentration of holdup in top vessel reaches the specified value. At this time, all of the vessels are isolated from the column, and the liquid in top vessel is drained away as the product. The holdups in other 2 vessels are moved upwards in turn. Then, the remaining filled vessels are connected to the column and the system repeats above-mentioned operations until the holdups in all vessels reach desired composition. During the experimental process, the new operation mode is easy to be operated, the reason of which is that the column is always run under constant total reflux. The experiment with regular batch column is conducted in the same column without vessels and distillate is collected in the product receiver, so the parameters of column are all same.

\section{Experimental results and discussion}

Two binary chemical systems, either of which is fairly ideal, methanol-ethanol and ethanol- 1-propanol, are studied in the experiment. Sample composition analysis is performed off-line by using an Agilent $6890 \mathrm{~N}$ Gas Chromatography equipped with an Agilent J\&W HP-FFAP capillary column $(30 \mathrm{~m} \times 0.53 \mathrm{~mm} \times 1 \mu \mathrm{m})$ and a Flame Ionization Detector (FID). The operation time to obtain the same amount of product with specification purity is set as the comparing parameter to evaluate the performances of different operations. Before the experiments are carried out, the regular batch distillation process is simulated to optimize the reflux ratio.

The experimental specifications and the results are shown in Table 4. It is easy to find out that the multivessel column has obvious advantage in saving time. Compared to the regular batch column, the reduction of operation time for the separation of methanol-ethanol and ethanol-1-propanol is approximately $28.46 \%$ and $27.08 \%$, respectively. The top concentration profile with time of the multivessel column and regular batch column for separation of two mixtures is presented in Figure 5. It demonstrates that the total reflux operation time for the first vessel is longer than that for the constant finite reflux operation. The result can be explained in terms of the negative effect of the liquid holdups in three vessels. With the higher amount of total holdup in column and vessels, the product concentration in the top vessel increases more slowly and requires more time to reach the specification value.

It is well known that the total reflux operation has the maximum fractionating capacity for the column with the same number of theoretical trays, while regular batch distillation under the reflux ratio of a constant value does not make full use of the separation ability of the packed column. As a result, the multivessel column shows great superiority under the same operating conditions

Table 4. Experimental conditions and results

\begin{tabular}{|c|c|c|c|c|}
\hline \multirow{2}{*}{$\begin{array}{l}\text { Material group } \\
\text { Operation mode }\end{array}$} & \multicolumn{2}{|c|}{ Methanol-Ethanol } & \multicolumn{2}{|c|}{ Ethanol-1-Propanol } \\
\hline & $\begin{array}{l}\text { Regular batch } \\
\text { column }\end{array}$ & Multivessel column & $\begin{array}{l}\text { Regular batch } \\
\text { column }\end{array}$ & Multivessel column \\
\hline Amount of feed $[\mathrm{mL}]$ & 1600 & 1600 & 1750 & 1750 \\
\hline Initial feed composition $\left[x_{F}\right]$ & 0.17 & 0.17 & 0.183 & 0.183 \\
\hline Reflux ratio & 10 & $\infty$ & 6 & $\infty$ \\
\hline Amount of product [mL] & 176.0 & 180.0 & 179.6 & 180.0 \\
\hline $\begin{array}{l}\text { Product composition } \\
\text { [mass fraction] }\end{array}$ & 0.847 & 0.852 & 0.933 & 0.951 \\
\hline Operation time [min] & 664 & 475 & 432 & 315 \\
\hline Time saving [min] & - & $\begin{array}{l}189 \\
(28.46 \%)\end{array}$ & - & $\begin{array}{l}117 \\
(27.08 \%)\end{array}$ \\
\hline
\end{tabular}




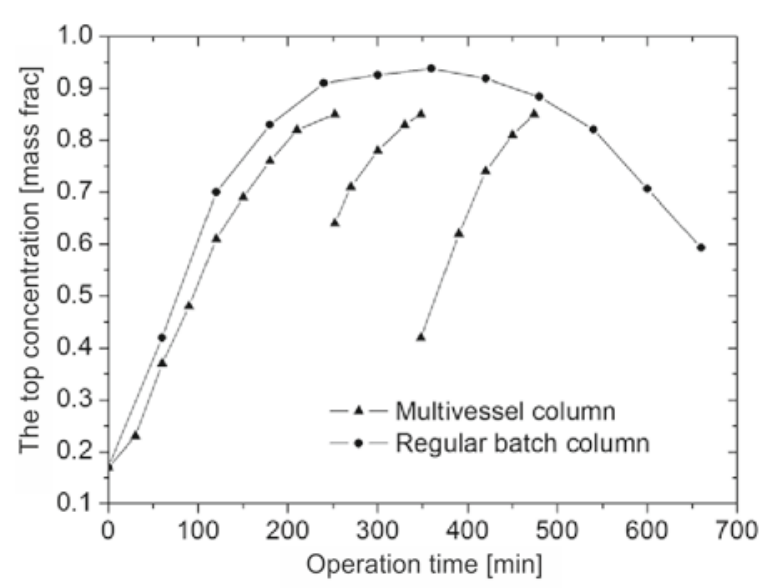

(a)

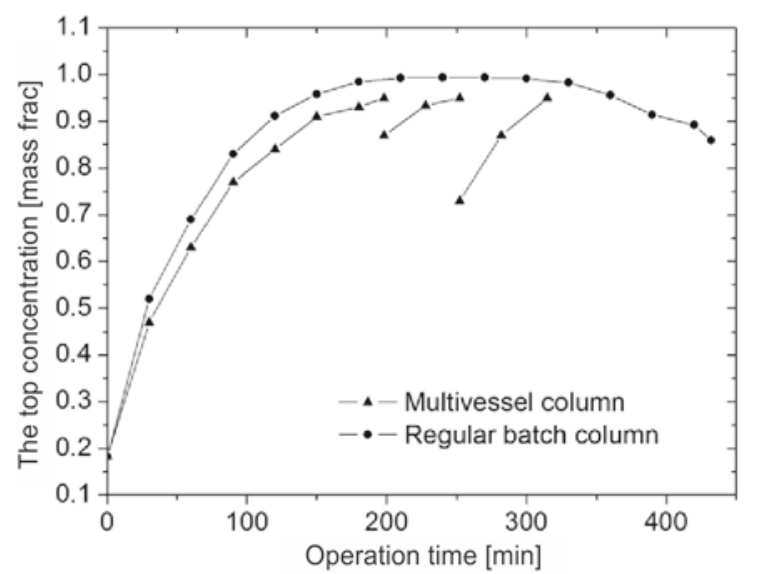

(b)

Figure 7. The top concentration profile with time of different column configurations (a) methanol and ethanol; (b) ethanol and 1-propanol

such as feed concentration, product concentration and relative volatility, etc.

The experiments show good agreement with the simulations, and confirm that the multivessel column with constant total reflux using in separation of binary system is easier to operate and provides new potential for the improvement of batch distillation.

\section{CONCLUSIONS}

In this paper, the multivessel column is proposed to separate a binary zeotropic mixture. For designing the multivessel column, two different configurations are developed. One is the conventional multivessel column with vapor bypass, and the other is the modified multivessel column without vapor bypass. By using the mechanical modeling technique the relative mathematic models are set up for dynamic simulations in MATLAB software.

Then, we present batch time requirements for the separation of binary zeotropic mixtures in two different multivessel columns, a non-cyclic two-vessel column and a regular batch column based on dynamic simulations. The first three columns are operated under total reflux and the regular batch column is operated under constant reflux ratio. The results show that multivessel configuration, either conventional or modified, has greater potential to save operation time than traditional configurations like the regular batch column and the non-cyclic two-vessel column, when a system is difficult to be separated. The modified multivessel column requires much less time than the conventional one, because the elimination of the vapor bypass improves the composition dynamics in the intermediate vessels. However, practical issues make the idea of eliminating the vapor bypass in the multivessel column configuration unattractive. Thus, the multivessel column with vapor bypass is recommended for the separation of a binary zeotropic system that is difficult to be separated.

Finally, a laboratory-scale multivessel column with the vapor stream bypassing the intermediate vessel is built. Two binary zeotropic systems, methanol-ethanol and ethanol-1-propanol, are studied in the experiment. The corresponding experiments confirm that the multivessel column with vapor bypass is feasible and easy to implement and operate, and it is of great practical importance in the pharmaceutical, fine and specialty chemicals industries.

\section{NOMENCLATURE}

$\alpha \quad-$ Relative volatility

$F \quad$ - Initial feed, mol

$H_{\mathrm{i}} \quad$ - Holdup on the $i$ th stage, mol

$H_{\mathrm{T}} \quad$ - Holdup in the top vessel of the non-cyclic two-vessel column, mol

$H_{\mathrm{V}} \quad$ - Holdup in each vessel of the multivessel column, mol

$H_{\mathrm{B}} \quad$ - Holdup in the reboiler of the multivessel column, mol

$L \quad$ - Liquid flow, $\mathrm{mol} / \mathrm{min}$

$L_{\mathrm{i}} \quad$ - Liquid flow leaving the $i$ th stage, $\mathrm{mol} / \mathrm{min}$

$n_{\mathrm{C}} \quad$ - Number of components

$n_{\mathrm{N}} \quad-$ Number of total stages

$n_{\mathrm{V}}-$ Number of vessels

$N_{1} \quad$ - Theoretical stage number of the section 1 in the multivessel column

$N_{2} \quad$ - Theoretical stage number of the section 2 in the multivessel column

$N_{3} \quad$ - Theoretical stage number of the section 3 in the multivessel column

$N_{\text {total }}$ - Total number of theoretical stage

$R \quad-$ Reflux ratio

Rec - Product recovery

$V \quad$ - Vapor flow, $\mathrm{mol} / \mathrm{min}$

$V_{\mathrm{i}} \quad$ - Vapor flow leaving the $i$ th stage, $\mathrm{mol} / \mathrm{min}$

$x_{\mathrm{i}, \mathrm{j}} \quad$ - Liquid concentration of the $j$ th component leaving the $i$ th stage, mole fraction

$x_{\mathrm{F}} \quad-$ Initial feed composition

$x_{\mathrm{p}} \quad-$ Product purity

$x_{\mathrm{V} 1}$ - Concentration of light component in Vessel 1

$x_{\mathrm{V} 2}$ - Concentration of light component in Vessel 2

$x_{\mathrm{V} 3}$ - Concentration of light component in Vessel 3

$x_{\mathrm{Bot}}$ - Concentration of light component in the reboiler

$y_{\mathrm{i}, \mathrm{j}} \quad$ - Vapor concentration of the $j$ th component leaving the $i$ th stage, mole fraction

\section{LITERATURE CITED}

1. Sørensen, E. \& Skogestad, S. (1996). Comparison of regular and inverted batch distillation, Chem. Eng. Sci. 51(22), 4949-4962. DOI: 10.1016/0009-2509(96)00287-4. 
2. Klein, A. \& Repke, J.U. (2009). Regular and inverted batch process structures for pressure swing distillation: a case study, Asia-Pac. J. Chem. Eng. 4(6), 893-904. DOI: 10.1002/apj.344.

3. Masoud, A.Z. \& Mujtaba, I.M. (2009). Effect of operating decisions on the design and energy consumption of inverted batch distillation column, Chem. Prod. Proc. Model., 4(1), Article 35. DOI: 10.2202/1934-2659.1275.

4. Davidyan, A.G., Kiva, V.N., Meski, G.A. \& Morari, M. (1994). Batch distillation in a column with a middle vessel, Chem. Eng. Sci. 49(18), 3033-3051. DOI: 10.1016/0009-2509(94) E0083-3.

5. Meski, G.A. \& Morari, M. (1995). Design and operation of a batch distillation column with a middle vessel, Comput. Chem. Eng. 19, 597-602. DOI: 10.1016/0098-1354(95)87100-4.

6. Barolo, M., Guarise, G.B., Rienzi, S.A., Trotta, A. \& Macchietto, S. (1996). Running batch distillation in a column with a middle vessel, Ind. Eng. Chem. Res. 35(12), 4612-4618. DOI: $10.1021 /$ ie $960268 s$.

7. Cui, X.B., Yang, Z.C., Shao, H.Q. \& Qu, H.M. (2001). Batch distillation in a column with a cold middle vessel for heat-sensitive compounds, Ind. Eng. Chem. Res. 40(3), 879-884. DOI: 10.1021/ie000491w.

8. Warter, M., Demicoli, D. \& Stichlmair, J. (2004). Operation of a batch distillation column with a middle vessel: experimental results for the separation of zeotropic and azeotropic mixtures, Chem. Eng. Process. 43(3), 263-272. DOI: 10.1016/ S0255-2701(03)00122-3.

9. Gruetzmann, S., Fieg, G. \& Kapala, T. (2006). Theoretical analysis and operating behaviour of a middle vessel batch distillation with cyclic operation, Chem. Eng. Process. 45(1), 46-54. DOI: 10.1016/j.cep.2005.05.005.

10. Gruetzmann, S. \& Fieg, G. (2008). Startup operation of middle-vessel batch distillation column: modeling and simulation, Ind. Eng. Chem. Res. 47(3), 813-824. DOI: 10.1021/ ie $070667 \mathrm{v}$.

11. Babu, G., Aditya, R. \& Jana, A.K. (2012). Economic feasibility of a novel energy efficient middle vessel batch distillation to reduce energy use, Energy 45(1), 626-633. DOI: 10.1016/j.energy.2012.07.035.

12. Edreder, E.A., Mujtaba, I.M. \& Emtir, M.M. (2012). Simulation of middle vessel batch reactive distillation column: application to hydrolysis of methyl lactate, Chem. Eng. Trans. 29, 595-600. DOI: 10.3303/CET1229100.

13. Monroy-Loperena, R. \& Alvarez-Ramírez, J. (2012). Dual composition control in continuous, middle-vessel distillation columns, with a draw stream in the middle vessel, Ind. Eng. Chem. Res. 51(12), 4624-4631. DOI: 10.1021/ie203018k.

14. Mori, H., Ito, C., Oda, A. \& Aragaki, T. (1999). Total reflux simulation of packed column distillation, J. Chem. Eng. Jpn. 32(1), 69-75. DOI: 10.1252/jcej.32.69.

15. Hegely, L. \& Lang, P. (2011). Comparison of closed and open operation modes of batch distillation, Chem. Eng. Trans. 25, 695-700. DOI: 10.3303/CET1125116.

16. Skouras, S. \& Skogestad, S. (2004). Time requirements for heteroazeotropic distillation in batch columns, Comput. Chem. Eng. 28(9), 1689-1700. DOI: 10.1016/j.compchemeng.2004.01.004

17. Skouras, S. \& Skogestad, S. (2004). Time (energy) requirements in closed batch distillation arrangements, Comput. Chem. Eng. 28(5), 829-837. DOI: 10.1016/j.compchemeng.2004.02.021.

18. Bai, P., Hua, C., Li, X. \& Yu, K.T. (2005). Cyclic total reflux batch distillation with two reflux drums, Chem. Eng. Sci. 60(21), 5845-5851. DOI: 10.1016/j.ces.2005.05.040.

19. Bai, P., Song, S., Sheng, M. \& Li, X. (2010). A dynamic modeling for cyclic total reflux batch distillation, Chinese. J. Chem. Eng. 18(4), 554-561. DOI: 10.1016/S1004-9541(10)60258-3.

20. Jiang, Z. \& Bai, P. (2011). Overhead concentration platform of total withdrawal operation in cyclic total reflux batch distillation, Chinese. J. Chem. Eng. 19(4), 598-602. DOI: 10.1016/S1004-9541(11)60028-1.
21. Bortolini, P. \& Guarise, G.B. (1970). A new practice of batch distillation, Quad. dell'Ing. Chim. Ital., 6(9), 150-157.

22. Treybal, R.E. (1970). A simple method for batch distillation, Chem. Eng. 77, 95-101.

23. Wittgens, B., Litto, R., Sørensen, E. \& Skogestad, S. (1996). Total reflux operation of multivessel batch distillation, Comput. Chem. Eng. 20, 1041-1046. DOI: 10.1016/00981354(96)00181-0.

24. Skogestad, S., Wittgens, B., Litto, R. \& Sørensen, E. (1997). Multivessel batch distillation, AIChE J. 43(4), 971-978. DOI: $10.1002 /$ aic.690430412.

25. Hasebe, S., Noda, M. \& Hashimoto, I. (1997). Optimal operation policy for multi-effect batch distillation system, Comput. Chem. Eng. 21, 1221-1226. DOI: 10.1016/S00981354(97)87669-7.

26. Furlonge, H., Pantelides, C. \& Sørensen, E. (1999). Optimal operation of multivessel batch distillation columns, AIChE J. 45(4), 781-801. DOI: 10.1002/aic.690450413.

27. Hasebe, S., Noda, M. \& Hashimoto, I. (1999). Optimal operation policy for total reflux and multi-effect batch distillation systems, Comput. Chem. Eng. 23(4), 523-532. DOI: 10.1016/S0098-1354(98)00290-7.

28. Wittgens, B. \& Skogestad, S. (2000). Closed operation of multivessel batch distillation: Experimental verification, $A I C h E$ J. 46(6), 1209-1217. DOI: 10.1002/aic.690460613.

29. Kurooka, T., Nishitani, H., Hasebe, S. \& Hashimoto, I. (2001). Energy conservation by multi-effect batch distillation system, J. Chem. Eng. Jpn. 34(9), 1141-1146. DOI: 10.1252/ jcej.34.1141.

30. Mahmud, M.T., Mujtaba, I.M. \& Emtir, M. (2008). Optimal design and operation of multivessel batch distillation column with fixed product demand and strict product specifications, Comp. Aid. Chem. Engin., 25(1), 253-258. DOI: 10.1016/ S1570-7946(08)80047-8.

31. Gruetzmann, S., Fieg, G. \& Skogestad, S. (2009). Experimental and theoretical studies on the start-up operation of a multivessel batch distillation column, Ind. Eng. Chem. Res. 48(11), 5336-5343. DOI: 10.1021/ie800962b.

32. Mujtaba, I. (2004). Batch Distillation. Design and operation. London, UK: Imperial College Press. 